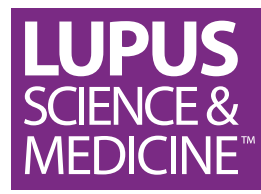

\title{
Clinical response beyond the Systemic Lupus Erythematosus Responder Index: post-hoc analysis of the BLISS-SC study
}

To cite: van Vollenhoven RF, Stohl W, Furie RA, et al. Clinical response beyond the Systemic Lupus Erythematosus Responder Index: post-hoc analysis of the BLISS-SC study. Lupus Science \& Medicine 2018;5:e000288. doi:10.1136/ lupus-2018-000288

Received 26 June 2018 Revised 16 August 2018 Accepted 20 August 2018

\section{Check for updates}

(C) Author(s) (or their employer(s)) 2018. Re-use permitted under CC BY. Published by BMJ.

For numbered affiliations see end of article.

Correspondence to Professor Ronald F van Vollenhoven; r.f. vanvollenhoven@amc.uva.nl

\section{ABSTRACT}

Objective The Systemic Lupus Erythematosus (SLE) Responder Index (SRI), developed as a primary outcome measure for use in clinical trials, captures improvement in SLE disease activity without concomitant worsening in disease manifestations. This study investigated the relationships between the SRI and clinical/laboratory correlates of SRI response in patients with SLE. Methods This was a post-hoc analysis of the phase III, double-blind, placebo-controlled study of subcutaneous BeLimumab in Subjects with Systemic lupus erythematosus - SubCutaneous (BLISS-SC). Patients were randomised to weekly belimumab $200 \mathrm{mg}$ subcutaneously or placebo, plus standard SLE therapy. Changes from baseline to week 52 in clinical and laboratory parameters were compared among SRI responders and nonresponders, irrespective of the treatment received. Results SRI responders ( $n=475)$ had significantly better $(p<0.0001)$ outcomes compared with non-responders ( $n=358)$, including (by definition) higher proportions achieving $\geq 4$-point improvement in Safety of Estrogens in Lupus Erythematosus National Assessment-SLE Disease Activity Index (100.0\% vs 2.0\%), no worsening in British Isles Lupus Assessment Group (BILAG; 0 new BILAG A or $\leq 1$ new BILAG B score; $100.0 \%$ vs $50.3 \%$ ) and no worsening ( $<0.3$-point increase) in Physician's Global Assessment score (100.0\% vs $49.7 \%)$. Among patients receiving $>7.5 \mathrm{mg} /$ day corticosteroids at baseline, significantly more SRI responders had reductions in prednisone dose to $\leq 7.5 \mathrm{mg} /$ day than non-responders. SR responders reported lower flare rates and improvements in serological markers and Functional Assessment of Chronic Illness Therapy-Fatigue score than non-responders.

Conclusion SRI response is associated with improvements in clinical and laboratory measures, strengthening its value as a clinically meaningful primary endpoint in clinical trials.

\section{INTRODUCTION}

Systemic lupus erythematosus (SLE) is a chronic autoimmune disease that is highly heterogeneous in its presentation. ${ }^{1}$ Accurate clinical assessment of SLE is imperative due to its diverse presentation and the fluctuations in disease symptoms and severity over time. ${ }^{2}$
Most SLE-specific measures of disease activity are used only in clinical trials and observational studies in specialised centres; they are not routinely used in everyday clinical practice, as they are not considered practical for busy clinical settings. ${ }^{3}$

The Systemic Lupus Erythematosus Responder Index (SRI) was developed as a primary outcome measure for use in clinical trials. ${ }^{4}$ It uses the Safety of Estrogens in Lupus Erythematosus National Assessment-SLE Disease Activity Index (SELENA-SLEDAI), British Isles Lupus Assessment Group (BILAG), and the Physician's Global Assessment (PGA) to measure changes in disease activity in patients with SLE. ${ }^{4}$ The SRI was designed to capture improvement in SLE disease activity without concomitant worsening in other disease manifestations. ${ }^{4}$ However, the perceived complexity of the SRI suggests its clinical meaningfulness in relation to measurements of SLE disease activity routinely used in 'real-world' clinical practice requires clarifying.

A post-hoc analysis of the phase III trials of intravenous belimumab (BLISS-52 and BLISS-76) assessed the association of the SRI response at week 52 with clinical and laboratory measures of SLE, patient-reported health-related quality of life (HRQoL) and fatigue among SRI responders and non-responders, irrespective of treatment. The results of this study suggested that an SRI response is associated with improved clinical, laboratory and patient-reported outcome measures in patients with SLE. ${ }^{5}$

BLISS-SC (BEL112341; NCT01484496), a phase III, randomised, 52-week trial was conducted to assess the efficacy and safety of subcutaneous belimumab in patients with SLE. ${ }^{6}$ In this post-hoc analysis, we investigated the relationships between SRI response and clinical and laboratory measures of SLE among patients in the BLISS-SC study 
to examine the clinical relevance of achieving an SRI response.

\section{MATERIALS AND METHODS}

\section{Study design and patients}

This was a post-hoc analysis of the phase III, multicentre, 52-week, placebo-controlled BLISS-SC study (BEL112341; NCT01484496), carried out at 207 sites in 31 countries, between November 2011 and September $2015 .{ }^{6}$

The study design and methods have been described in detail previously ${ }^{6}$ and are summarised here. Patients were required to have a diagnosis of SLE according to the American College of Rheumatology criteria, ${ }^{7}$ with positive antinuclear antibodies (ANA) and/or anti-double-stranded DNA (anti-dsDNA), a SELENA-SLEDAI score $\geq 8$, and to be receiving stable standard of care therapy (SoC) for $\geq 30$ days. Patients with severe lupus kidney disease (proteinuria $>6 \mathrm{~g} / 24$ hours or equivalent using spot urine protein to creatinine ratio, or serum creatinine $>2.5 \mathrm{mg} / \mathrm{dL}$ ), severe active nephritis (requiring acute therapy not permitted in the study protocol (eg, intravenous cyclophosphamide), or have required haemodialysis, or high-dose prednisone or equivalent within 90 days prior to baseline), or severe central nervous system (CNS) lupus (including seizures, psychosis, organic brain syndrome, cerebrovascular accident, cerebritis or CNS vasculitis) requiring therapeutic intervention within 60 days of baseline were excluded. Patients were randomised 2:1 to weekly belimumab $200 \mathrm{mg}$ subcutaneously or placebo administered using a prefilled syringe, plus SoC. The primary endpoint of BLISS-SC was the SRI response rate at week 52. An SRI responder was defined as a patient who had $\geq 4$-point reduction in SELENA-SLEDAI score, no new BILAG A or $\leq 1$ new BILAG $\mathrm{B}$ domain scores, and no deterioration ( $<0.3$-point increase) from baseline in the $\mathrm{PGA}^{4}$ Severe flare rates, reductions or increases in prednisone use, and changes in fatigue, as measured by the Functional Assessment of Chronic Illness Therapy (FACIT)-Fatigue scale, are not included within the SRI, but were included as additional endpoints. Patients were considered non-responders if they did not meet the SRI response criteria, withdrew before week 52 or received protocol-prohibited medications.

All patients provided written informed consent prior to enrolment. The study was conducted in accordance with the Declaration of Helsinki $2008 .^{8}$

\section{Endpoints and assessments}

Changes from baseline to week 52 in clinical and laboratory parameters among SRI responders and non-responders were compared. Parameters analysed included change from baseline to week 52 in SELENA-SLEDAI, BILAG, PGA and corticosteroid use (prednisone equivalent dose). The incidence of any (mild, moderate or severe) Systemic Lupus Erythematosus Flare Index (SFI) and severe SFI flares were also compared. Health outcomes were assessed using FACIT-Fatigue scores. ${ }^{9} 10$ Biomarker assessments included normalisation of anti-dsDNA, and serum complement levels (C3 and C4) and mean per cent change from baseline in B cell subsets (CD20+; naïve CD19+/CD20+/ CD27-; activated CD19+/CD20+/CD69+; memory CD19+/ CD20+/CD27+; plasmacytoid CD19+/CD20+/CD138+; plasma CD19+/CD20-/CD138+; SLE subset CD19+/ $\mathrm{CD} 38 \mathrm{~b}+/ \mathrm{CD} 27 \mathrm{~b}+\mathrm{lymph}$; transitional $\mathrm{CD} 19+/ \mathrm{CD} 24 \mathrm{~b}+/$ $\mathrm{CD} 38 \mathrm{~b}+/ \mathrm{CD} 27-$ ). B cell counts (with the exception of CD20+ B cells) were normalised to CD19+ B cell counts.

\section{Data analyses}

In this post-hoc analysis, SRI responders and non-responders were compared regardless of the treatment received. With the exception of the biomarker analyses, all analyses were conducted using the last observation carried forward.

Components of the SRI and changes in corticosteroid dose from baseline to week 52 were compared using logistic regression models with a standard least squares (LS) method. Changes in SELENA-SLEDAI, BILAG, PGA, corticosteroid dose, FACIT-Fatigue and biomarkers were assessed using an analysis of covariance model comparing SRI responders and non-responders at week 52. The time to first severe SFI flare was compared between SRI responders and non-responders using a Cox proportional hazards model. Shifts in anti-dsDNA and complement levels were evaluated using Fisher's exact test.

The analyses were performed using Statistical Analysis Software (SAS) V.9.3.

\section{RESULTS}

\section{Patient population}

The intent-to-treat population comprised 836 patients; 3 patients did not have a PGA assessment at baseline, so they could not be categorised as SRI responders or non-responders and were therefore excluded from these post-hoc analyses. In total there were 475 SRI responders and 358 SRI non-responders. Overall, $677(81.0 \%)$ patients completed the study, and 159 (19.0\%) patients withdrew prior to week 52 ; common reasons for withdrawal were adverse events $(7.8 \%)$ and patient request (3.2\%). Baseline characteristics were balanced across SRI responders and non-responders (table 1). The majority of patients were female (94.4\%), and the mean (standard deviation [SD]) age was 38.6 (12.28) years. The mean (SD) SELENA-SLEDAI scores at baseline were 10.9 (3.08; SRI responders) and 9.8 (3.09; SRI non-responders) (table 1).

\section{SRI components: SELENA-SLEDAI, BILAG and PGA}

At week 52, all SRI responders (by definition) had $\geq 4$-point reduction in SELENA-SLEDAI score compared with $2.0 \%$ of non-responders $(\mathrm{p}<0.0001$; (figure 1A). SRI responders consistently had a significantly greater LS mean (standard error [SE]) improvement in SELENA-SLEDAI score than non-responders from baseline to week 52 (week 52: -7.01 $(0.225)$ vs -1.31 (0.217), respectively; $\mathrm{p}<0.0001)$ (figure $1 \mathrm{~B}$ ).

Significantly more SRI responders ( $100 \%$, by definition) had no worsening attributable to SLE in multiple organ systems as measured by BILAG (no new BILAG A or $\leq 1$ new BILAG B score) compared with non-responders (50.3\%; $\mathrm{p}<0.0001$ ) at week 52 (figure 1A). From baseline to week 52, 
Table 1 Patient demographics and characteristics

\begin{tabular}{|c|c|c|c|}
\hline & SRI non-responders & SRI responders & Overall \\
\hline ITT population & $(n=358)$ & $(n=475)$ & $(n=833)$ \\
\hline Female, n (\%) & 335 (93.6) & $451(94.9)$ & $786(94.4)$ \\
\hline Median SLE disease duration, years (range) & $5.0(0-38)$ & $4.0(0-33)$ & $4.4(0-38)$ \\
\hline Mean baseline SELENA-SLEDAI score (SD) & $9.8(3.09)$ & $10.9(3.08)$ & $10.4(3.14)$ \\
\hline SELENA-SLEDAI score $\geq 10, \mathrm{n}(\%)$ & $189(52.8)$ & $331(69.7)$ & $520(62.4)$ \\
\hline \multicolumn{4}{|l|}{ BILAG organ domain involvement*, n (\%) } \\
\hline$\geq 1 \mathrm{~A}$ or $2 \mathrm{~B}$ & $246(68.7)$ & $351(73.9)$ & $597(71.7)$ \\
\hline$\geq 1 \mathrm{~A}$ & $61(17.0)$ & $77(16.2)$ & $138(16.6)$ \\
\hline$\geq 1 \mathrm{~B}$ & $325(90.8)$ & $429(90.3)$ & $754(90.5)$ \\
\hline $0 \mathrm{mg} / \mathrm{day}$ & $59(16.5)$ & $54(11.4)$ & $113(13.6)$ \\
\hline$>0$ to $\leq 7.5 \mathrm{mg} /$ day & $85(23.7)$ & $133(28.0)$ & $218(26.2)$ \\
\hline$>7.5 \mathrm{mg} /$ day & $214(59.8)$ & $288(60.6)$ & $502(60.3)$ \\
\hline$\geq 1 \mathrm{SFI}$ flare, $\mathrm{n}(\%) \dagger$ & $66(18.4)$ & $83(17.5)$ & $149(17.9)$ \\
\hline$\geq 1$ severe SFI flare, $n(\%) \dagger$ & $8(2.2)$ & $4(0.8)$ & $12(1.4)$ \\
\hline Anti-dsDNA-positive ( $\geq 30 \mathrm{IU} / \mathrm{mL}), \mathrm{n}(\%)$ & $250(69.8)$ & $345(72.6)$ & $595(71.4)$ \\
\hline Low C3 (<90 mg/dL), n (\%) & $144(40.2)$ & $210(44.2)$ & $354(42.5)$ \\
\hline Low C4 (<10 mg/dL), n (\%) & $94(26.3)$ & $121(25.5)$ & $215(25.8)$ \\
\hline
\end{tabular}

*Patients may be included in more than one category.

†During the screening period (day -35 to day 0 ).

Anti-dsDNA, anti-double-stranded DNA; BILAG, British Isles Lupus Assessment Group; ITT, intent-to-treat (all randomised patients treated with $\geq 1$ dose of study treatment); PGA, Physician's Global Assessment; SELENA-SLEDAI, Safety of Estrogens in Lupus Erythematosus National Assessment-Systemic Lupus Erythematosus Disease Activity Index; SFI, Systemic Lupus Erythematosus Flare Index; SRI, Systemic Lupus Erythematosus Responder Index.

significantly greater improvements were seen in all BILAG and SELENA-SLEDAI organ domains for SRI responders compared with non-responders (table 2).

All SRI responders (by definition) had no worsening $(<0.3$-point increase) in their overall condition as assessed by the PGA score compared with $49.7 \%$ of non-responders $(\mathrm{p}<0.0001)$ from baseline to week 52 (figure 1A). SRI responders had a significantly greater LS mean (SE) improvement in PGA score from baseline to week 52 compared with non-responders $(-1.02(0.036)$ vs -0.39 $(0.034)$, respectively; $\mathrm{p}<0.0001$ ) (figure $1 \mathrm{C}$ ).

\section{Additional components: prednisone use, flares, biomarkers and FACIT-Fatigue \\ Prednisone use}

At baseline, $60.6 \%$ of SRI responders and $59.8 \%$ of nonresponders received $>7.5 \mathrm{mg} /$ day prednisone (or equivalent; table 1). Among patients receiving $>7.5 \mathrm{mg} /$ day prednisone at baseline, significantly more SRI responders $(68 / 288 ; 23.6 \%)$ were able to reduce prednisone dose to $\leq 7.5 \mathrm{mg}$ /day at week 52 than non-responders $(23 / 214$; $10.7 \%$; odds ratio[OR] 2.49, 95\% confidence interval [CI] 1.49 to $4.15 ; \mathrm{p}=0.0005$ ) (figure 2A). There was a significant difference between the two groups from week 40 to week 52. Fewer SRI responders who received prednisone $\leq 7.5$ $\mathrm{mg}$ /day at baseline had increases in dose to $>7.5 \mathrm{mg}$ /day at week 52 than non-responders $(6 / 187(3.2 \%)$ vs $20 / 144$ (13.9\%); OR $0.20,95 \%$ CI 0.08 to $0.52 ; \mathrm{p}=0.0009)$. There was a significant difference between the two groups from week 4 to week 52, with the exception of week 8 (figure 2B).

Flares

Significantly fewer SRI responders (272/475; 57.3\%) than non-responders $(256 / 358 ; 71.5 \% ; \mathrm{p}<0.0001)$ had mild, moderate or severe flares over the 52-week period (figure 2C). The median time (25th, 75th percentile) to first flare was longer in SRI responders (223 (85, noncalculable) days) than non-responders $(113(57,330)$ days; $\mathrm{p}<0.0001)$. Significantly more SRI responders $(459 / 475$; $96.6 \%)$ than non-responders $(260 / 353 ; 73.7 \%$; $<<0.0001)$ 
A

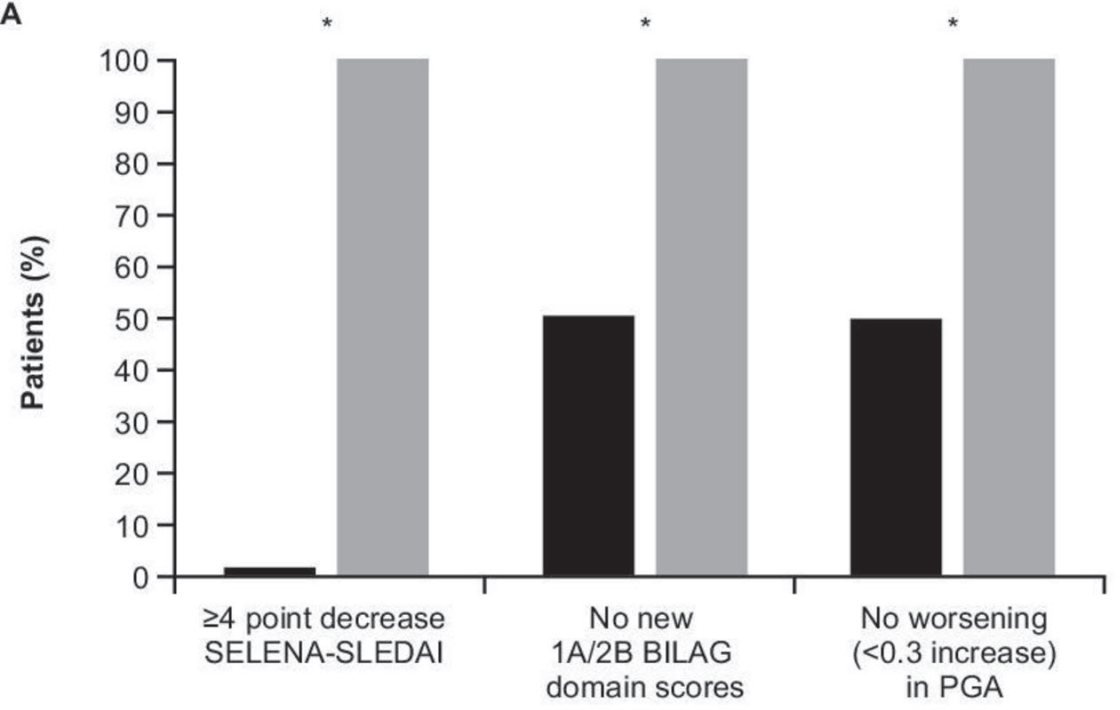

B

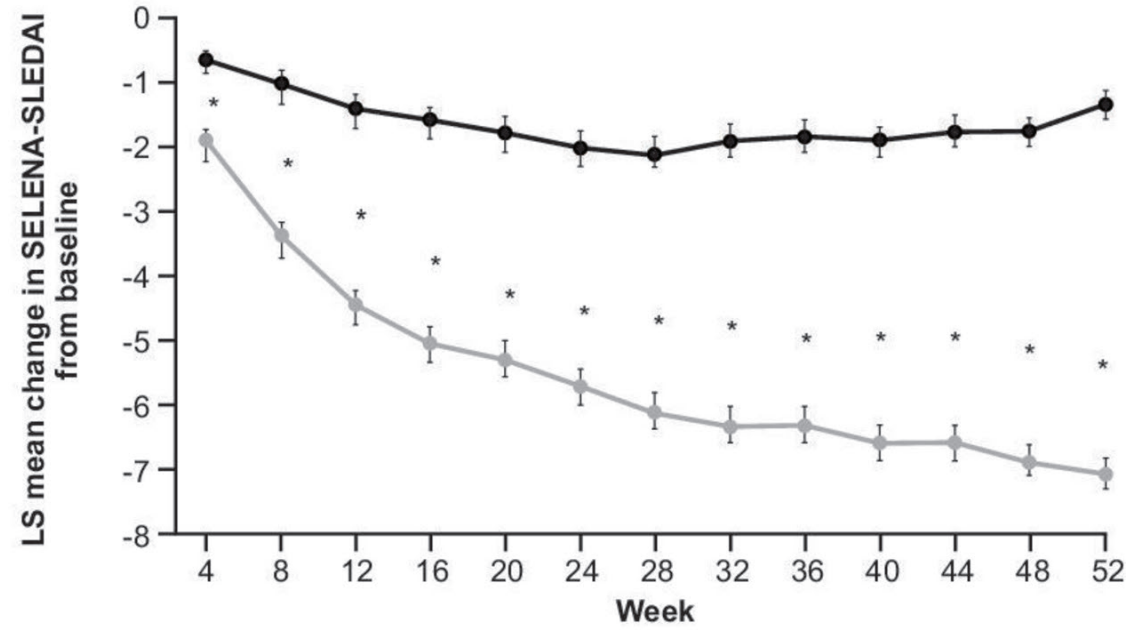

C

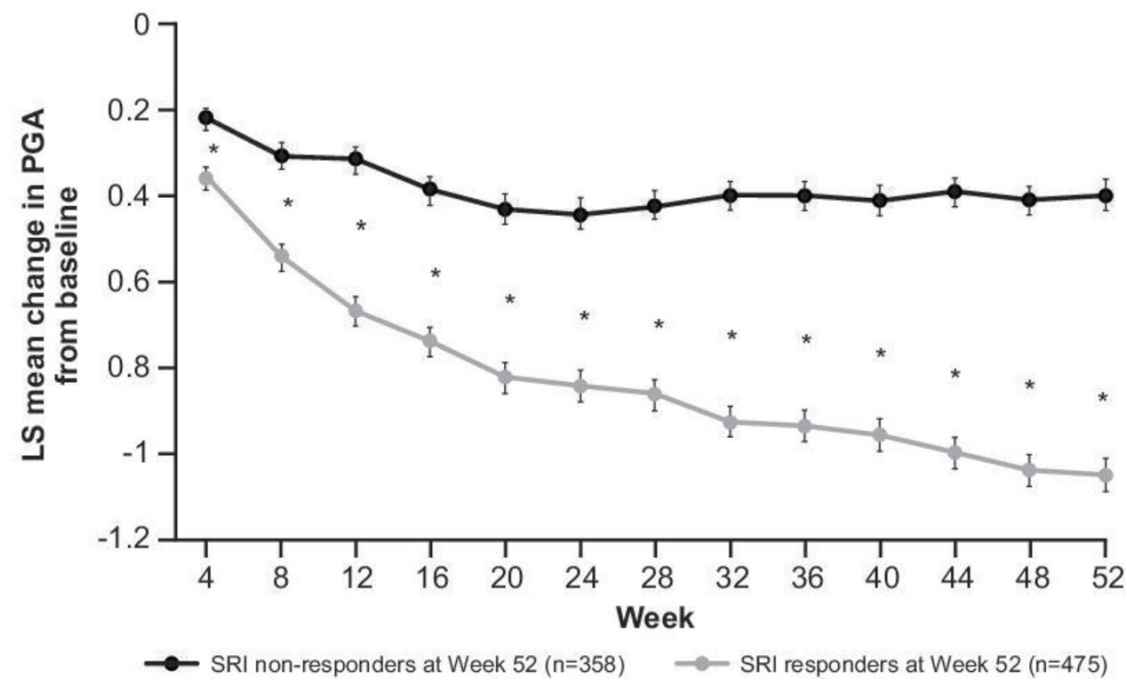

Figure 1 (A) SRI 4 components (week 52); LS mean change from baseline in (B) SELENA-SLEDAI and (C) PGA. * $p<0.0001$ SRI responders versus non-responders. BILAG, British Isles Lupus Assessment Group; LS, least squares; PGA, Physician's Global Assessment; SELENA-SLEDAI, Safety of Estrogens in Lupus Erythematosus National Assessment-SLE Disease Activity Index; SRI, Systemic Lupus Erythematosus Responder Index. 
Clinical trials and drug discovery

Table 2 Improvement in BILAG (A or B) and SELENA-SLEDAl by organ domain/system

SRI non-responders

$(n=358)$
Treatment difference, SR

responders vs

SRI responders $(n=475)$ non-responders $(95 \% \mathrm{Cl})$
$P$ values*

\section{BILAG organ domain}

General

\begin{tabular}{|c|c|c|c|c|}
\hline A or B score at baselinet, $n$ & 28 & 40 & & \\
\hline Improvement at week 52‡, n (\%) & $15(53.6)$ & $39(97.5)$ & 43.9 (24.83 to 63.02$)$ & $<0.0001$ \\
\hline \multicolumn{5}{|l|}{ Cardiovascular and respiratory } \\
\hline A or B score at baselinet, $n$ & 12 & 8 & & \\
\hline Improvement at week 52‡, n (\%) & $3(25.0)$ & $8(100.0)$ & 75.0 (50.50 to 99.50$)$ & 0.0014 \\
\hline \multicolumn{5}{|l|}{ Haematology } \\
\hline A or B score at baselinet, $n$ & 67 & 70 & & \\
\hline Improvement at week 52‡, n (\%) & $12(17.9)$ & $32(45.7)$ & 27.8 (12.95 to 42.65$)$ & 0.0005 \\
\hline \multicolumn{5}{|l|}{ Mucocutaneous } \\
\hline A or B score at baselinet, $n$ & 235 & 329 & & \\
\hline Improvement at week 52ł, n (\%) & $54(23.0)$ & $235(71.4)$ & 48.5 (41.19 to 55.71$)$ & $<0.0001$ \\
\hline \multicolumn{5}{|l|}{ Musculoskeletal } \\
\hline A or B score at baselinet, $n$ & 251 & 369 & & \\
\hline Improvement at week 52ł, n (\%) & $50(19.9)$ & $338(91.6)$ & 71.7 (65.98 to 77.37$)$ & $<0.0001$ \\
\hline \multicolumn{5}{|l|}{ Neurological } \\
\hline A or B score at baselinet, $n$ & 1 & 3 & & \\
\hline Improvement at week 52‡, n \% & $1(100.0)$ & $3(100.0)$ & $0.0(0.00$ to 0.00$)$ & \\
\hline \multicolumn{5}{|l|}{ Renal } \\
\hline A or B score at baselinet, $n$ & 39 & 44 & & \\
\hline Improvement at week 52‡, n (\%) & $12(30.8)$ & $37(84.1)$ & 53.3 (35.25 to 71.39$)$ & $<0.0001$ \\
\hline \multicolumn{5}{|l|}{ Vasculitis } \\
\hline A or B score at baselinet, $n$ & 29 & 42 & & \\
\hline Improvement at week 52‡, n (\%) & $7(24.1)$ & $37(88.1)$ & 63.9 (45.56 to 82.36$)$ & $<0.0001$ \\
\hline \multicolumn{5}{|c|}{ SELENA-SLEDAI organ system improvement, by category, $\mathbf{n}(\%)$} \\
\hline \multicolumn{5}{|l|}{ CNS total } \\
\hline Baseline involvement $\dagger, \mathrm{n}$ & 4 & 5 & & \\
\hline Improvement at week 52§, n \%) & $0(0.0)$ & $5(100.0)$ & $100.0(100.00$ to 100.00$)$ & 0.0079 \\
\hline \multicolumn{5}{|l|}{ Cardiovascular and respiratory total } \\
\hline Baseline involvement†, $n$ & 19 & 28 & & \\
\hline Improvement at week 52§, n \%) & $5(26.3)$ & $25(89.3)$ & 63.0 (40.09 to 85.85$)$ & $<0.0001$ \\
\hline \multicolumn{5}{|l|}{ Haematological total } \\
\hline Baseline involvement $\dagger, \mathrm{n}$ & 27 & 46 & & \\
\hline Improvement at week 52§, n (\%) & $6(22.2)$ & $24(52.2)$ & $30.0(8.64$ to 51.27$)$ & 0.0146 \\
\hline \multicolumn{5}{|l|}{ Immunological total } \\
\hline Baseline involvement $\dagger, \mathrm{n}$ & 267 & 369 & & \\
\hline Improvement at week 52§, n (\%) & $18(6.7)$ & $143(38.8)$ & 32.0 (26.20 to 37.82$)$ & $<0.0001$ \\
\hline \multicolumn{5}{|l|}{ Mucocutaneous total } \\
\hline Baseline involvement $\dagger, \mathrm{n}$ & 303 & 431 & & \\
\hline Week 52§, n (\%) & $92(30.4)$ & $355(82.4)$ & $52.0(45.70$ to 58.31$)$ & $<0.0001$ \\
\hline \multicolumn{5}{|l|}{ Musculoskeletal total } \\
\hline Baseline involvement $\dagger, \mathrm{n}$ & 256 & 398 & & \\
\hline Improvement at week 52§, n (\%) & $28(10.9)$ & $363(91.2)$ & 80.3 (75.54 to 85.00$)$ & $<0.0001$ \\
\hline
\end{tabular}

*Fisher's exact test.

†Number used as denominator for percentages.

fPatients who have an $A$ at baseline and change to a $B, C$ or $D$, or patients with a $B$ at baseline who change to a $C$ or $D$, are considered to have improvement.

§An improvement is defined as a decrease (compared with baseline) in SELENA-SLEDAI score within the same organ system.

BILAG, British Isles Lupus Assessment Group; CNS, central nervous system; SELENA-SLEDAI, Safety of Estrogens in Lupus Erythematosus National Assessment- Systemic Lupus Erythematosus Disease Activity Index; SRI, Systemic Lupus Erythematosus Responder Index. 

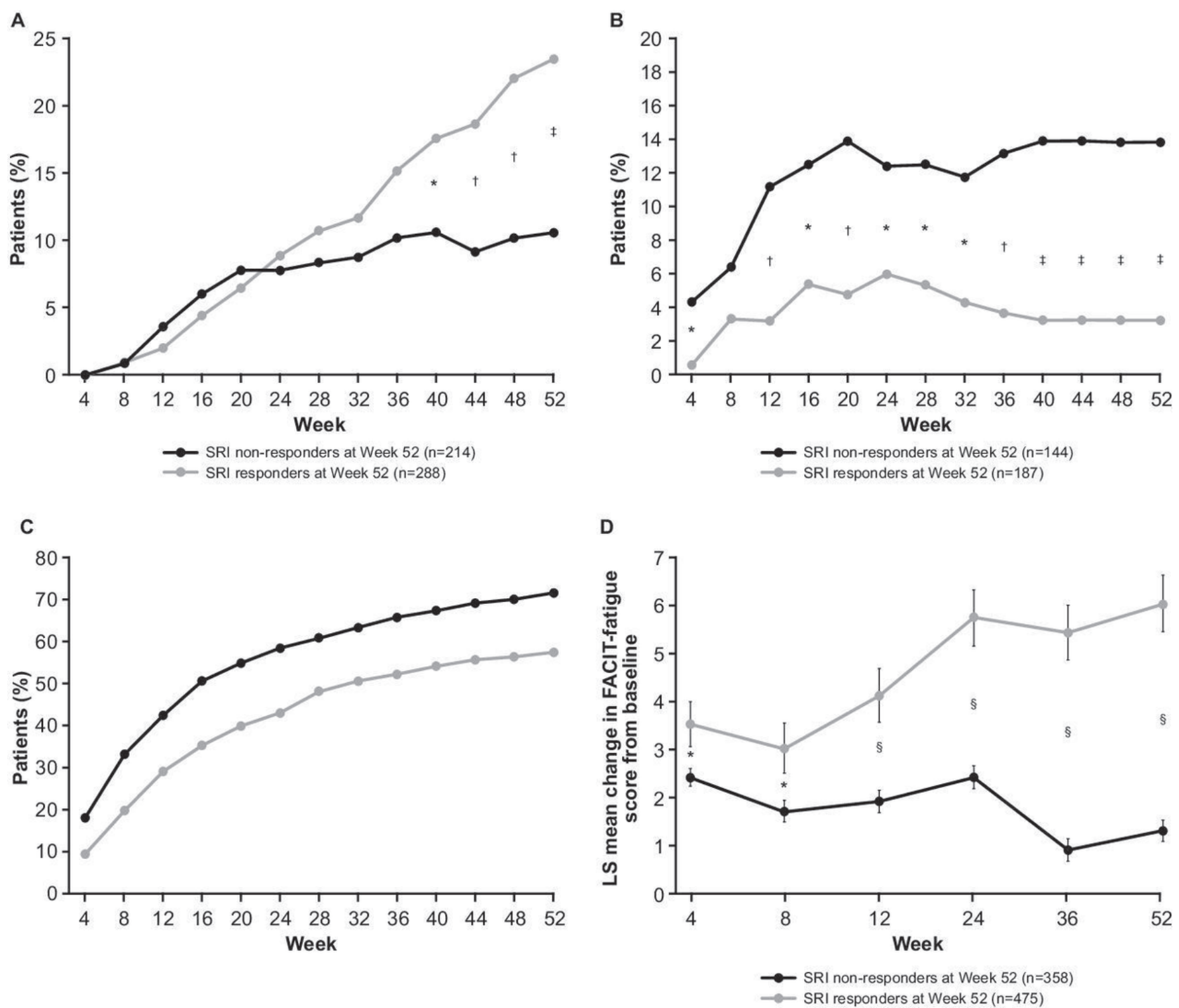

Figure 2 (A) Decrease in prednisone dose from $>7.5 \mathrm{mg} /$ day at baseline to $\leq 7.5 \mathrm{mg} /$ day; (B) increase in prednisone dose from $\leq 7.5 \mathrm{mg} /$ day at baseline to $>7.5 \mathrm{mg} /$ day; (C) cumulative incidence of SFI flares; (D) LS mean change from baseline in FACITFatigue score. ${ }^{*} p<0.05 ; \nmid p<0.01 ; \ddagger p<0.001 ; \S p \leq 0.0001$. FACIT-Fatigue, Functional Assessment of Chronic Illness TherapyFatigue; LS, least squares; SFI, Systemic Lupus Erythematosus Flare Index; SRI, Systemic Lupus Erythematosus Responder Index.

had no severe flares; therefore, severe flares occurred in $3.4 \%$ of SRI responders vs $26.3 \%$ of non-responders.

\section{Biomarkers}

At baseline, $71.4 \%$ of patients were anti-dsDNA-positive $(\geq 30 \mathrm{IU} / \mathrm{mL}), 42.5 \%$ had low C3 levels $(<90 \mathrm{mg} / \mathrm{dL})$ and $25.8 \%$ had low C4 levels $(<10 \mathrm{mg} / \mathrm{dL}$ ) (table 1$)$. Significantly more SRI responders $(71 / 336 ; 21.1 \%)$ than non-responders $(9 / 140 ; 6.4 \%)$ had normalised antidsDNA at week $52(\mathrm{p}<0.0001)$ (figure $3 \mathrm{~A})$. The median (25th, 75th percentile) percentage change in anti-dsDNA antibodies was $-46.7 \%(-68.3,-10.7)$ for SRI responders compared with $-16.7 \%(-49.5,5.5 ; \mathrm{p}=0.0171)$ for non-responders, from baseline to week 52. Among patients with low C3 at baseline, a greater proportion of SRI responders shifted to normal/high C3 levels at week 52
$(87 / 205 ; 42.4 \%)$ compared with non-responders $(14 / 78$; $17.9 \% ; \mathrm{p}<0.0001$ ) (figure 3B). From baseline to week 52, the median (25th, 75 th percentile) percentage change in $\mathrm{C} 3$ across all patients was $6.3 \%(-6.8,21.8)$ for SRI responders compared with $-2.1 \%(-10.9,12.2 ; \mathrm{p}=0.0024)$ for non-responders. In patients with low $\mathrm{C} 4$ at baseline, more SRI responders shifted to normal/high levels at week $52(59 / 120 ; 49.2 \%)$ compared with non-responders $(14 / 45 ; 31.1 \%$; $p=0.0523)$ (figure 3C). From baseline to week 52, the median (25th, 75 th percentile) percentage change in C4 across all patients was $16.7 \%(0,44.4)$ for SRI responders compared with $5.9 \%(-14.3,24.1 ; \mathrm{p}=0.0540)$ for non-responders.

Similar decreases in the levels of B cell subsets (CD20+; naïve $\mathrm{CD} 19+/ \mathrm{CD} 20+/ \mathrm{CD} 27-$; activated $\mathrm{CD} 19+/ \mathrm{CD} 20+$ / 
A

$\longrightarrow$ SRI non-responders: anti dsDNA positive to negative

- - SRI responders: anti dsDNA positive to negative

--A-- SRI non-responders: anti dsDNA negative to positive

--K-- SRI responders: anti dsDNA negative to positive

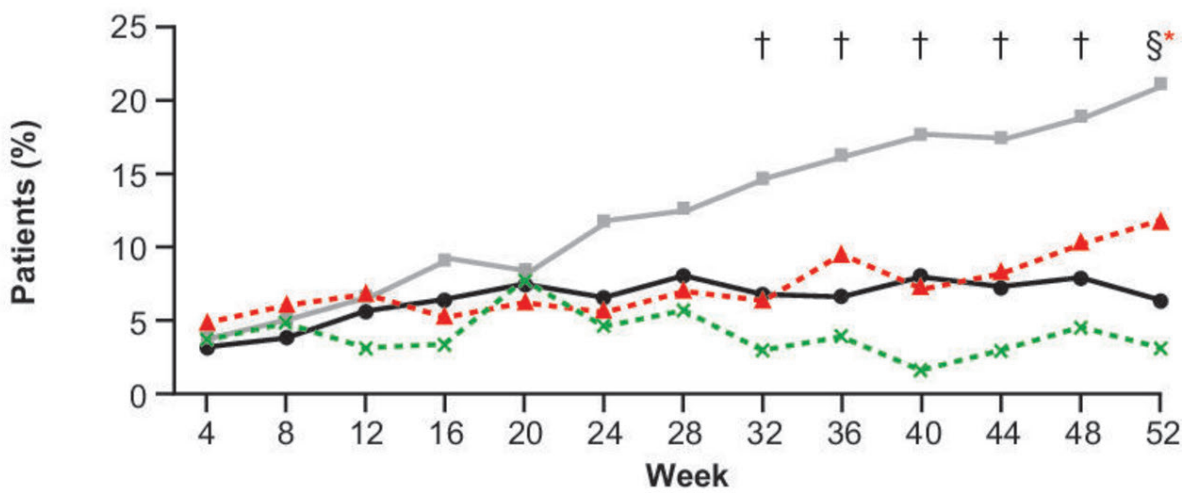

B $\quad \longrightarrow$ SRI non-responders: C3 low to normal/high

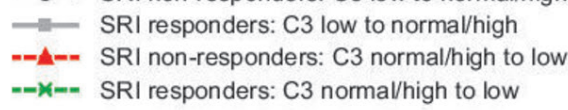

--M-- SRI responders: C3 normal/high to low

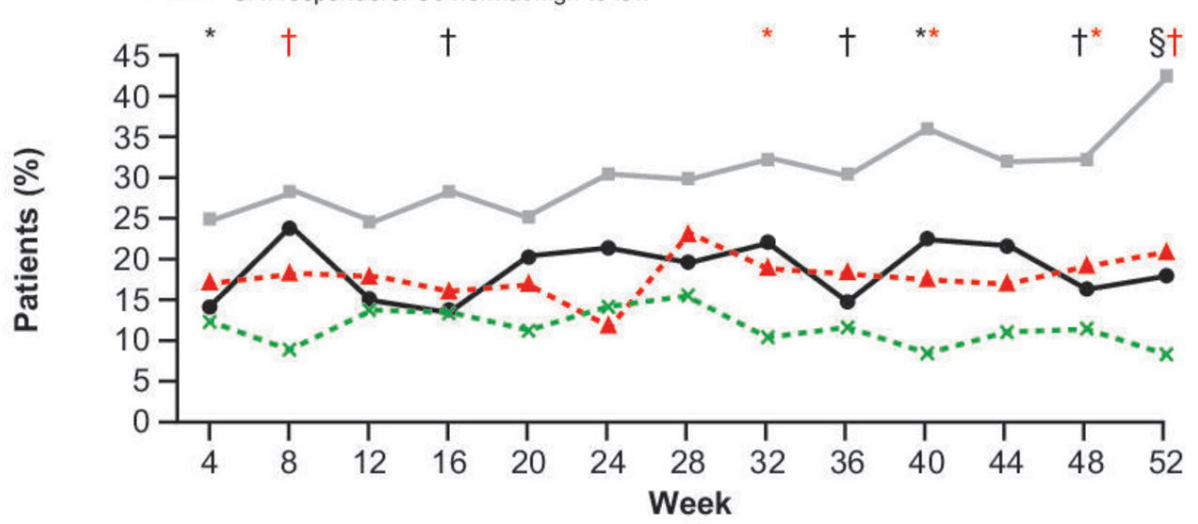

C
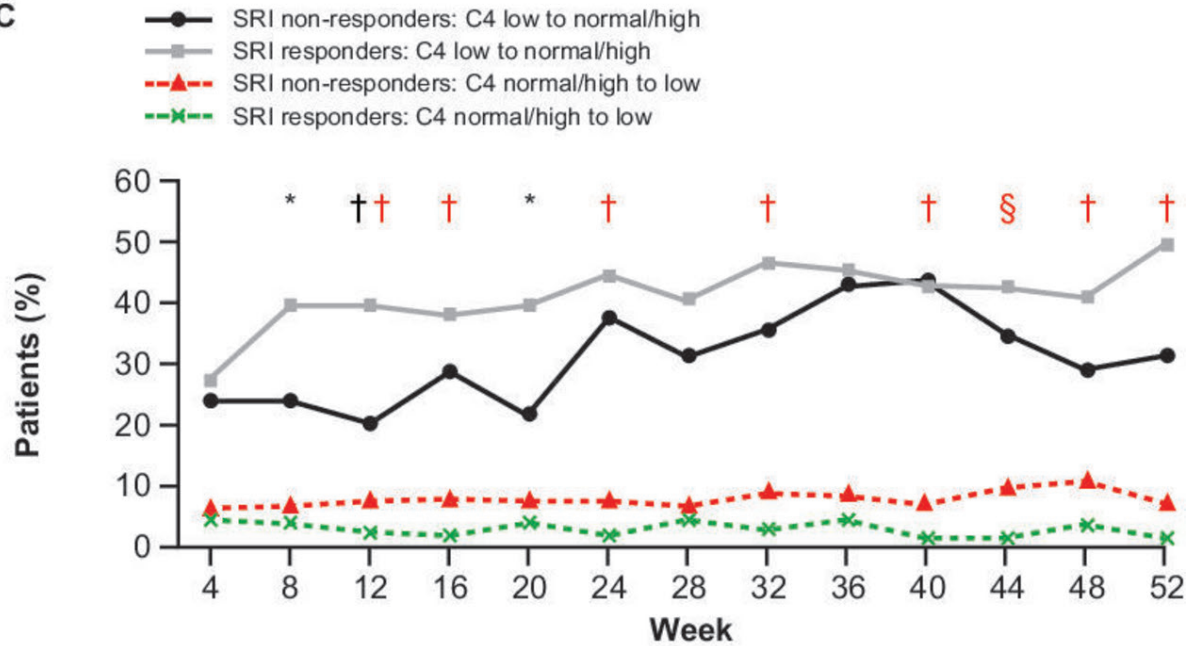

Figure 3 Change in biomarkers over time: (A) anti-dsDNA (IU/mL), (B) C3 (mg/dL) and (C) C4 (mg/dL).

Anti-dsDNA, anti-double-stranded DNA; SRI, Systemic Lupus Erythematosus Responder Index. $* \mathrm{p}<0.05 \mathrm{SRI}$ responders versus non-responders (anti-dsDNA positive to negative; C3/C4 low to normal/high); $* \mathrm{p}<0.05 \mathrm{SRI}$ responders versus non-responders (anti-dsDNA negative to positive; C3/C4 normal/high to low); $\mathrm{t}<0.01 \mathrm{SRI}$ responders versus non-responders (anti-dsDNA positive to negative; C3/C4 low to normal/high); $t \mathrm{p}<0.01 \mathrm{SRI}$ responders versus non-responders (anti-dsDNA negative to positive; C3/C4 normal/high to low); $\neq \mathrm{p}<0.001 \mathrm{SRI}$ responders versus non-responders (anti-dsDNA positive to negative; C3/C4 low to normal/high); $\neq \mathrm{p}<0.001 \mathrm{SRI}$ responders versus non-responders (anti-dsDNA negative to positive; C3/C4 normal/high to low); $\$ p \leq 0.0001 \mathrm{SRI}$ responders versus non-responders (anti-dsDNA positive to negative; C3/C4 low to normal/high); $\$ \mathrm{p} \leq 0.0001$ $\mathrm{SRI}$ responders versus non-responders (anti-dsDNA negative to positive; C3/C4 normal/high to low). 
CD69+; memory CD19+/CD20+/CD27+; plasmacytoid CD19+/CD20+/CD138+; plasma CD19+/CD20-/CD138+; SLE subset CD19+/CD38b+/CD27b+ lymph; transitional $\mathrm{CD} 19+/ \mathrm{CD} 24 \mathrm{~b}+/ \mathrm{CD} 38 \mathrm{~b}+/ \mathrm{CD} 27-)$ were observed in SRI responders and non-responders from baseline to week 52 (data not shown).

\section{FACIT-Fatigue}

The mean (SE) percentage change in FACIT-Fatigue score from baseline to week 52 was greater for SRI responders $(35.6 \%(4.02))$ than non-responders $(18.7 \%$ (3.53); $\mathrm{p}<0.0001$; figure 2D). A significantly higher proportion of SRI responders had a minimal clinically important difference improvement in FACIT-Fatigue score of $\geq 4$ points at week 52 compared with non-responders $(53.9 \%$ vs $25.3 \%$; $\mathrm{p}<0.0001)$.

\section{DISCUSSION}

As SLE is a heterogeneous disease that impacts multiple organ systems, ${ }^{12}$ the SRI was designed to distinguish global response from non-response, based not only on improvement in SLE disease activity but on an absence of worsening of disease manifestations. ${ }^{4}$ It has been used in the four key phase III trials of belimumab plus SoC for the treatment of SLE: BLISS-52, BLISS-76, BLISS-SC and the recently completed study of intravenous belimumab in North East Asia. ${ }^{611-13}$ In all four trials the proportions of patients with an SRI response were significantly higher in the belimumab group compared with placebo. ${ }^{6} 1112$ Furthermore, a post-hoc analysis of the BLISS-52 and BLISS-76 trials demonstrated that SRI responders had greater improvements in clinical, laboratory and HRQoL measures compared with non-responders. ${ }^{5}$ In this post-hoc analysis of data from the BLISS-SC study, we investigated the relationships between SRI response and clinical and laboratory measures of SLE that are more commonly used in routine practice in order to examine the clinical meaningfulness of SRI response.

Overall, SRI responders had statistically significant better outcomes in multiple parameters compared with non-responders, including improvements in the individual SRI components (SELENA-SLEDAI, BILAG and PGA scores). These improvements observed within the individual SRI components demonstrate how the simpler individual components correlate effectively with the more complex SRI composite index. Reductions were also observed in non-SRI components, including reductions in corticosteroid dose, lower flare rates, improvements in serological markers and improvements in FACIT-Fatigue score. Improvement in these non-SRI components demonstrates the relevance of the SRI, an index routinely used within clinical trials, in reflecting measures that are more commonly used in clinical practice. Our findings are in line with those of a previous post-hoc analysis of the BLISS-52 and BLISS-76 studies of intravenous belimumab that found that SRI responders had greater improvements in clinical, serological and HRQoL measures of SLE disease activity compared with non-responders. ${ }^{5}$ For example, in this study $57.3 \%$ of SRI responders and $71.5 \%$ of non-responders had SFI flares $(\mathrm{p}<0.0001)$, compared with $69.9 \%$ and $82.7 \%$, respectively, in the intravenous belimumab post-hoc analysis $(\mathrm{p}<0.0001) .{ }^{5}$ Both of these studies also demonstrated that SRI responders had clinically significant greater improvements in BILAG and SELENA-SLEDAI organ domains than non-responders. ${ }^{5}$ In this study, greater improvements in all organ domains were observed in SRI responders compared with non-responders; however, it should be noted that in some cases the numbers of patients in the analyses were small.

Many studies have demonstrated that anti-dsDNA antibodies and low complement levels are associated with increased SLE disease severity. ${ }^{14-18}$ In the present study, an SRI response was associated with increases in C3 and/or C4 levels and decreases in anti-dsDNA antibody titres. There were no significant differences in changes in the levels of B cell subsets between SRI responders and non-responders. A pooled analysis of the BLISS-52 and BLISS-76 trials showed that belimumab, compared with placebo, was associated with significant reductions in CD20+ B cells and multiple B cell and plasma cell subsets, including naïve and activated $\mathrm{B}$ cells, while preserving the memory $\mathrm{B}$ cell subset ${ }^{19}$; however, there were no significant differences in the reductions of B cells in SRI responders versus non-responders. ${ }^{5}$ Similarly, in the current analysis, there were patients in both the SRI responder and non-responder groups who received belimumab, which may explain why there were no significant differences in changes in the levels of B cell subsets between the two groups.

Fatigue is one of the commonly reported symptoms among patients with SLE, and it considerably impacts patients' lives. ${ }^{20}$ The mean improvements in FACIT-Fatigue scores reported by SRI responders were greater than in non-responders ( $35.6 \%$ vs $8.7 \%$, respectively). The changes from baseline exceeded the minimal clinically important difference of $\geq 4$ points $^{910}$ at week 52 in over twice as many SRI responders compared with non-responders $(53.9 \%$ vs $25.3 \%$, respectively).

Interpretation of these results is limited by the post-hoc and observational nature of the analyses. Examination of the clinical trial population based on the primary endpoint eliminates the randomised balance of baseline characteristics in the treatment groups. Further, the inclusion criteria did not permit inclusion of patients with SELENA-SLEDAI scores $<8$ at screening, nor did they permit entry of patients with severe lupus kidney disease, severe active nephritis or active CNS lupus disease; thus, no conclusions can be made about these subgroups. Despite these limitations, the study used a large and robust data set to demonstrate the significance of an SRI response in terms that are relevant to clinicians. 
In conclusion, patients who were SRI responders, regardless of treatment, demonstrated improvements in numerous clinical and serological measures of disease activity compared with non-responders. This post-hoc analysis provides evidence that the SRI response represents a clinically meaningful outcome that can be used during clinical trials for patients with active, autoantibody-positive SLE.

\section{Author affiliations}

${ }^{1}$ Department of Rheumatology, Amsterdam Rheumatology and Immunology Center ARC, Amsterdam, The Netherlands

${ }^{2}$ University of Southern California Keck School of Medicine, Los Angeles, California, USA

${ }^{3}$ Northwell Health, Great Neck, New York, USA

${ }^{4}$ GSK, Clinical Development - Immuno-Inflammation, Rockville, Maryland, USA (at the time of the study)

${ }^{5}$ GSK, Clinical Development - Immuno-Inflammation, Philadelphia, Pennsylvania, USA

${ }^{6}$ GSK, Immuno-Inflammation and Future Pipeline, Collegeville, Pennsylvania, USA

${ }^{7}$ GSK, Biostatistics, Immuno-Inflammation, Collegeville, Pennsylvania, USA

${ }^{8}$ GSK, Medical Affairs, Research Triangle Park, North Carolina, USA

${ }^{9}$ GSK, Medical Affairs - Immuno-Inflammation, Research Triangle Park, North

Carolina, USA (at the time of the study)

${ }^{10}$ GSK, Medical Affairs, Rockville, Maryland, USA

Acknowledgements Editorial support (in the form of writing assistance, including development of the initial draft, assembling tables and figures, collating authors comments, grammatical editing and referencing) was provided by Katie White, PhD, and Shweta Vadnerkar, PhD, at Fishawack Indicia Ltd, UK, and was funded by GSK.

Contributors RFvV, DB and DG were involved in the study design, and in data analysis and interpretation. WS was involved in the acquisition of data and in data analysis and interpretation. RAF was involved in the study design, acquisition of data, and in data analysis and interpretation. NLF, JGG, MK, BFP, WJE and TG-R were involved in data analysis and interpretation. All authors contributed to the writing of this manuscript and approved the final version.

Funding This study was funded by GlaxoSmithKline (GSK; study number: BEL112341).

Competing interests RFvV has received consulting fees and/or honoraria from AbbVie, Biotest, Bristol-Myers Squibb, Crescendo, GSK, Janssen, Lilly, Merck, Pfizer, Roche, UCB and Vertex; research grants from AbbVie, Bristol-Myers Squibb, GSK, Pfizer, Roche and UCB; and is Editor-in-chief of Lupus Science \& Medicine. WS has received consulting fees from Akros Pharma, Janssen and Sanofi, and clinical trials support from GSK and Pfizer. RAF has received consulting fees, research grants and clinical trials support from GSK. NLF, WJE and DG were employees of GSK and held shares in GSK at the time of the study. JGG, DB, MK, BFP and TG-R are employees of GSK and hold shares in the company.

Patient consent All patients provided written informed consent prior to enrollment. Ethics approval The study and all protocols were approved by the Institutional Review Board and the study was conducted in accordance with the Declaration of Helsinki 2008.

Provenance and peer review Not commissioned; externally peer reviewed. Data sharing statement Anonymized individual participant data and study documents can be requested for further research from "http://www.clinicalstudydat arequest.com"

Open access This is an open access article distributed in accordance with the Creative Commons Attribution 4.0 Unported (CC BY 4.0) license, which permits others to copy, redistribute, remix, transform and build upon this work for any purpose, provided the original work is properly cited, a link to the licence is given, and indication of whether changes were made. See: http://creativecommons.org/ licenses/by/4.0/

\section{REFERENCES}

1. Kaul A, Gordon C, Crow MK, et al. Systemic lupus erythematosus. Nat Rev Dis Primers 2016;2:16039.

2. Lam GK, Petri M. Assessment of systemic lupus erythematosus. Clin Exp Rheumatol 2005;23(5 Suppl 39):S120-32.

3. Castrejón I, Tani C, Jolly M, et al. Indices to assess patients with systemic lupus erythematosus in clinical trials, long-term observational studies, and clinical care. Clin Exp Rheumatol 2014;32(5 Suppl 85):S-85-95.

4. Furie RA, Petri MA, Wallace DJ, et al. Novel evidence-based systemic lupus erythematosus responder index. Arthritis Rheum 2009;61:1143-51.

5. Furie R, Petri MA, Strand V, et al. Clinical, laboratory and healthrelated quality of life correlates of Systemic Lupus Erythematosus Responder Index response: a post hoc analysis of the phase 3 belimumab trials. Lupus Sci Med 2014;1:e000031.

6. Stohl W, Schwarting A, Okada M, et al. Efficacy and safety of subcutaneous belimumab in systemic lupus erythematosus: $A$ fifty-two-week randomized, double-blind, placebo-controlled study. Arthritis Rheumatol 2017;69:1016-27.

7. Hochberg MC. Updating the american college of rheumatology revised criteria for the classification of systemic lupus erythematosus. Arthritis Rheum 1997;40:1725.

8. World Medical Association, 2008. "WMA Declaration of Helsinki ethical principles for medical research involving human subjects.". Available from: http://www.wma.net/en/30publications/10policies/b3/ index.html

9. Lai JS, Beaumont JL, Ogale S, et al. Validation of the functional assessment of chronic illness therapy-fatigue scale in patients with moderately to severely active systemic lupus erythematosus, participating in a clinical trial. J Rheumatol 2011;38:672-9.

10. Cella D, Yount $S$, Sorensen $M$, et al. Validation of the functional assessment of chronic illness therapy fatigue scale relative to other instrumentation in patients with rheumatoid arthritis. J Rheumatol 2005;32:811-9.

11. Furie R, Petri M, Zamani O, et al. A phase III, randomized, placebocontrolled study of belimumab, a monoclonal antibody that inhibits B lymphocyte stimulator, in patients with systemic lupus erythematosus. Arthritis Rheum 2011;63:3918-30.

12. Navarra SV, Guzmán RM, Gallacher AE, et al. Efficacy and safety of belimumab in patients with active systemic lupus erythematosus: a randomised, placebo-controlled, phase 3 trial. Lancet 2011;377:721-31.

13. Zhang FBS, Bass D, Chu M. A pivotal phase III, randomized, placebo-controlled study of belimumab in patients with systemic lupus erythematosus located in China, Japan, and South Korea. Arthritis Rheumatol 2016;68.

14. Biesen R, Dähnrich C, Rosemann A, et al. Anti-dsDNA-NcX ELISA: dsDNA-loaded nucleosomes improve diagnosis and monitoring of disease activity in systemic lupus erythematosus. Arthritis Res Ther 2011;13:R26.

15. Merrill JT, Buyon JP. The role of biomarkers in the assessment of lupus. Best Pract Res Clin Rheumatol 2005;19:709-26.

16. Schur PH, Sandson J. Immunologic factors and clinical activity in systemic lupus erythematosus. N Engl J Med 1968;278:533-8.

17. Strand V, Chu AD. Measuring outcomes in systemic lupus erythematosus clinical trials. Expert Rev Pharmacoecon Outcomes Res 2011;11:455-68.

18. Thumboo J, Strand V. Health-related quality of life in patients with systemic lupus erythematosus: an update. Ann Acad Med Singapore 2007;36:115-22.

19. Stohl W, Hiepe F, Latinis KM, et al. Belimumab reduces autoantibodies, normalizes low complement levels, and reduces select $B$ cell populations in patients with systemic lupus erythematosus. Arthritis Rheum 2012;64:2328-37.

20. Tench CM, McCurdie I, White PD, et al. The prevalence and associations of fatigue in systemic lupus erythematosus. Rheumatology 2000;39:1249-54. 